\title{
SIMULAÇÃO DO TRANSPORTE DE GASES EM NANOESTRUTURAS POROSAS DE CARBONO
}

\author{
R. A. PAGNUSSATTI, J. KLEINPAUL, A. R. MUNIZ \\ Universidade Federal do Rio Grande do Sul, Departamento de Engenharia Química \\ E-mail para contato: rafaela@enq.ufrgs.br
}

\begin{abstract}
RESUMO - A busca de nanoestruturas porosas com alta estabilidade mecânica e propriedades versáteis é de grande interesse prático. Neste trabalho, avaliamos o potencial de aplicação de fulerenos porosos e nanoestruturas derivadas introduzidas recentemente na literatura em processos de separação de gases. Simulações de dinâmica molecular clássica são aplicadas para calcular propriedades de transporte de gases nessas estruturas, com o objetivo de analisar o desempenho destas como peneiras moleculares. Estas simulações permitem determinar as taxas de transporte de diversos gases $\left(\mathrm{CH}_{4}, \mathrm{CO}, \mathrm{O}_{2}, \mathrm{~N}_{2}, \mathrm{H}_{2}\right)$, através das nanoestruturas porosas, e a partir destas, as seletividades correspondentes para diferentes pares de substâncias de interesse prático $\left(\mathrm{CO} / \mathrm{H}_{2}, \mathrm{CH}_{4} / \mathrm{H}_{2}, \mathrm{O}_{2} / \mathrm{N}_{2} \ldots\right)$. As propriedades estimadas mostram que esses materiais podem atuar como membranas de alta seletividade, com grande potencial de aplicação na purificação de correntes gasosas em processos industriais.
\end{abstract}

\section{INTRODUÇÃO}

Materiais nanoporosos são materiais que tipicamente apresentam poros de diâmetro inferiores a $100 \mathrm{~nm}$, sendo os exemplos mais conhecidos as zeólitas, peneiras moleculares, carvão ativado, entre outros (Lu e Zhao, 2004). Suas principais propriedades são a leveza, uma elevada razão área/volume, e a possibilidade de apresentar uma distribuição de diâmetros uniforme. Estes encontram várias aplicações tecnológicas, como em separação de gases, adsorção, catálise, armazenamento de energia, e sensores. Estes materiais devem apresentar estabilidade mecânica e química, e deseja-se no processo de síntese, gerar materiais com diâmetros e características de superfície customizadas para determinada aplicação. Há uma busca contínua no desenvolvimento de novos materiais que atendam as especificações necessárias para aplicações inovadoras.

Foi introduzida recentemente uma classe de nanoestruturas de carbono em forma de gaiola gerada a partir do grafeno poroso e grafeno bifenileno (BPC), chamada de fulerenos porosos (Paupitz et al., 2014).Estes são estáveis até altas temperaturas e apresentam interessantes propriedades eletrônicas e para armazenamento/encapsulamento de moléculas. Este estudo apesar de ser teórico, sugeriu possibilidades para a síntese experimental, tal que estudos experimentais serão conduzidos para verificar a existência e viabilidade destes materiais. Estes materiais podem servir como bloco fundamental na construção de nanoestruturas porosas maiores, através da fusão de diversos destes fulerenos, que pode ser obtido através de aplicação de temperatura e pressão, funcionalização química entre outros processos.

A formação de estruturas bi e tridimensionais com porosidades variadas geradas a partir da 


\section{Congresso Brasileiro de Engenharia Química \\ em Iniciação Científica \\ UFSCar - São Carlos - SP \\ 16 a 19 de Julho de 2017}

CONGRESSO BRASILEIRO DE ENGENHARIA

coalescência de fulerenos porosos foi proposta em um estudo anterior do nosso grupo (Kleinpaul et al., 2016). As propriedades mecânicas destas nanoestruturas foram estimadas, mostrando que elas apresentam elevada resistência a tração, similar a nanoestruturas de carbono convencionais. Exemplos destas estruturas geradas são mostrados na Figura 1.

Figura 1 - Exemplos de estruturas geradas pela coalescência de fulerenos porosos. Em (a) temse uma estrutura unidimensional, equivalente a um nanofio, e em (b) e (c) duas estruturas planares, arranjadas em um lattice quadrado e de tal forma que poros de diferentes dimensões estejam presentes.

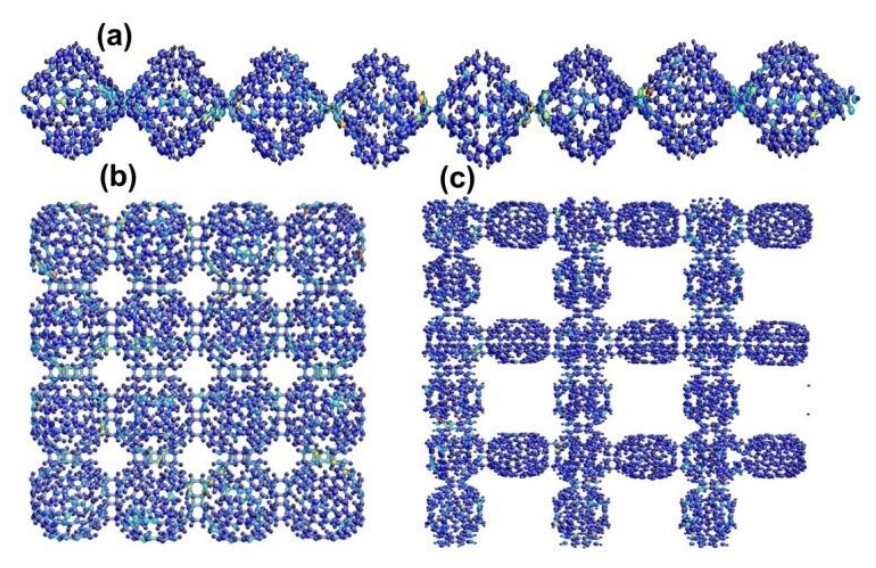

Neste trabalho avaliamos o desempenho dessas estruturas quando aplicadas em processos de separação de gases, devido ao fato de combinarem características que são desejáveis em materiais que atuam nesses processos, como a presença de poros manométricos com distribuição de diâmetros uniforme, leveza, e alta resistência mecânica e térmica. Simulações foram realizadas para calcular as taxas de transferência de diferentes gases e as seletividades para alguns pares destes.

\section{METODOLOGIA}

Para o cálculo das taxas de transporte dos gases nos materiais nanoporosos, foram empregadas simulações de dinâmica molecular (MD) clássica, usando o pacote LAMMPS (Plimpton, 1995). Simulações MD consistem na resolução das equações do movimento para $N$ corpos clássicos (correspondente aos $N$ átomos do sistema) que interagem de acordo com um potencial interatômico. Os potenciais utilizados para descrição das interações interatômicas foram o potencial de LennardJones, que representa as interações de van der Waals, e o potencial de Coulomb, que prevê as forças de interação eletrostáticas. Os parâmetros de Lennard-Jones utilizados para os gases e as cargas utilizadas foram obtidas no trabalho de Jiao e Xu (2015), e os parâmetros de interação para o carbono sólido foram extraídos do trabalho de Jorgensen et al. (1996) e Steele et al. (1990). Condições de contorno periódicas foram aplicadas nas direções de interesse de forma a representar um sistema infinito.

Membranas com diâmetro característico de poro de 5,7 Å foram criadas empilhando de dois a quatro dos planos de fulerenos porosos representados pela Figura 1 (b) (apresentando espessuras de 14 a $42 \AA$ ). A Figura 2(a) e 2(b) mostram a membrana formada por dois planos empilhados. Para as simulações de separação e gases, foram criados dois reservatórios, um preenchido com as moléculas de um gás e outro vazio, separados pela membrana, como 
mostrado na Figura 2(c). Realizou-se a contagem das moléculas que atravessam a membrana em função do tempo, determinando-se a taxa de difusão para os gases $\mathrm{H}_{2}, \mathrm{CO}, \mathrm{CH}_{4}, \mathrm{O}_{2}$ e $\mathrm{N}_{2}$ e diferentes seletividades. As simulações foram conduzidas usando um passo de integração de 1 fs, em um intervalo de até $10 \mathrm{~ns}$, sendo a temperatura mantida em $300 \mathrm{~K}$ sendo controlada pelo método de Nosé-Hoover. As moléculas são mantidas rígidas durante a simulação usando o algoritmo SHAKE.

Figura 2 - Membrana formada por fulerenos porosos estudada. Em (a) tem-se uma vista superior e em (b) uma vista lateral da membrana. Em (c) tem-se uma representação da caixa de simulação composta pelo reservatório preenchido pelas moléculas, o reservatório vazio, com a membrana de dupla camada separando os dois compartimentos.

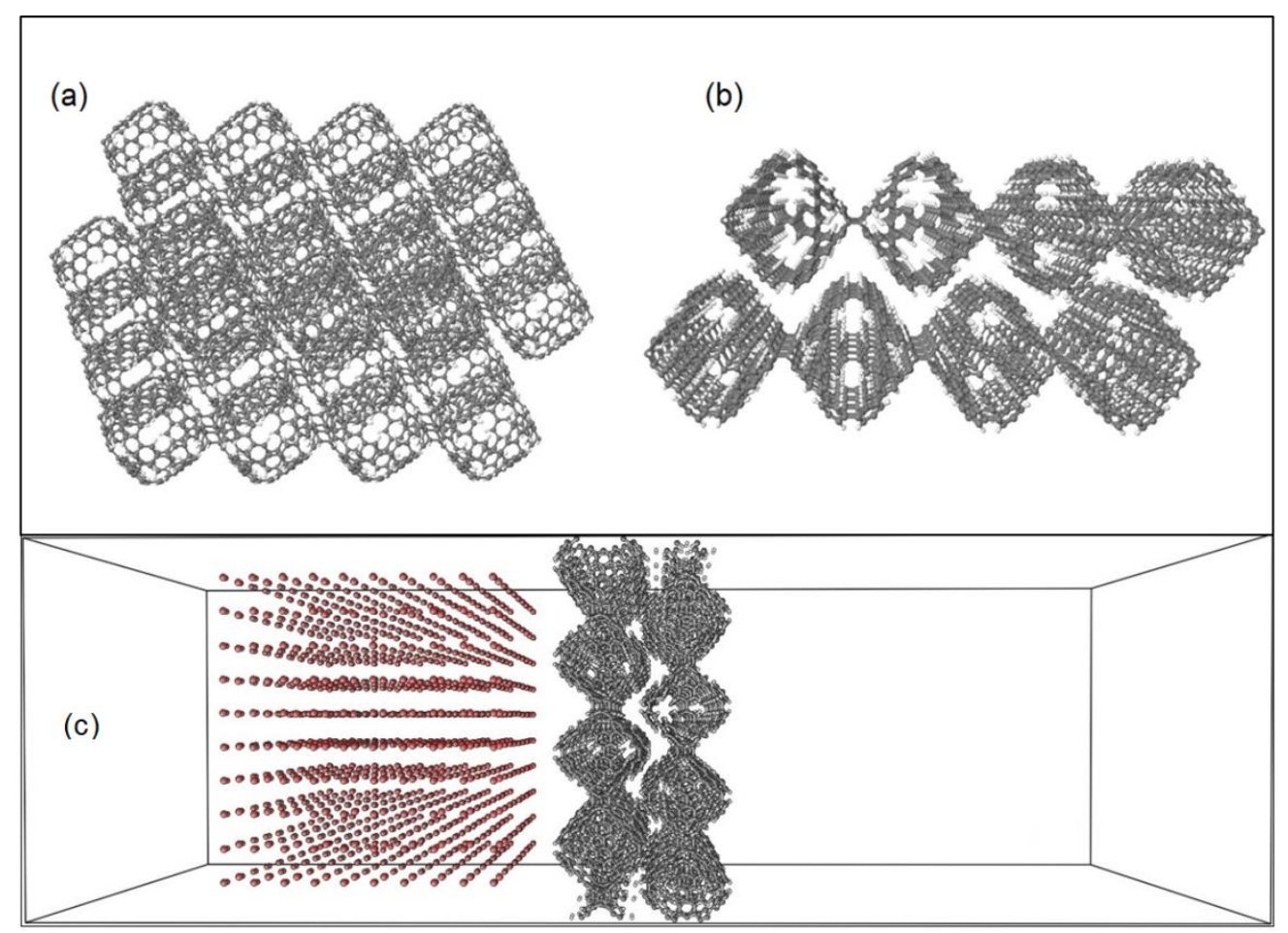

\section{RESULTADOS}

Foram conduzidas as simulações do processo de separação para os diferentes gases, em um total de até cinco simulações independentes para cada gás em cada membrana. A Figura 3 mostra um exemplo de resultado gerado na simulação de difusão do $\mathrm{CO}$ pela membrana, ilustrando as condições em três instantes de tempo distintos. Através do procedimento de contagem, foi possível gerar gráficos do número de moléculas que atravessaram a membrana (N) em função do tempo. A partir desses gráficos calculou-se a taxa inicial de difusão dos gases realizando uma regressão linear das curvas nesta região, quando há a maior diferença de concentração entre os dois lados da membrana. A Figura 4 exemplifica algumas curvas obtidas e o intervalo utilizado para fazer as regressões. A taxa de difusão (moléculas por tempo) é definida como a derivada da curva nesta região inicial:

$$
\operatorname{Taxa}=d N / d t
$$


Figura 3 - Estados observados em três instantes de tempo diferentes de uma simulação de difusão do CO pela membrana.
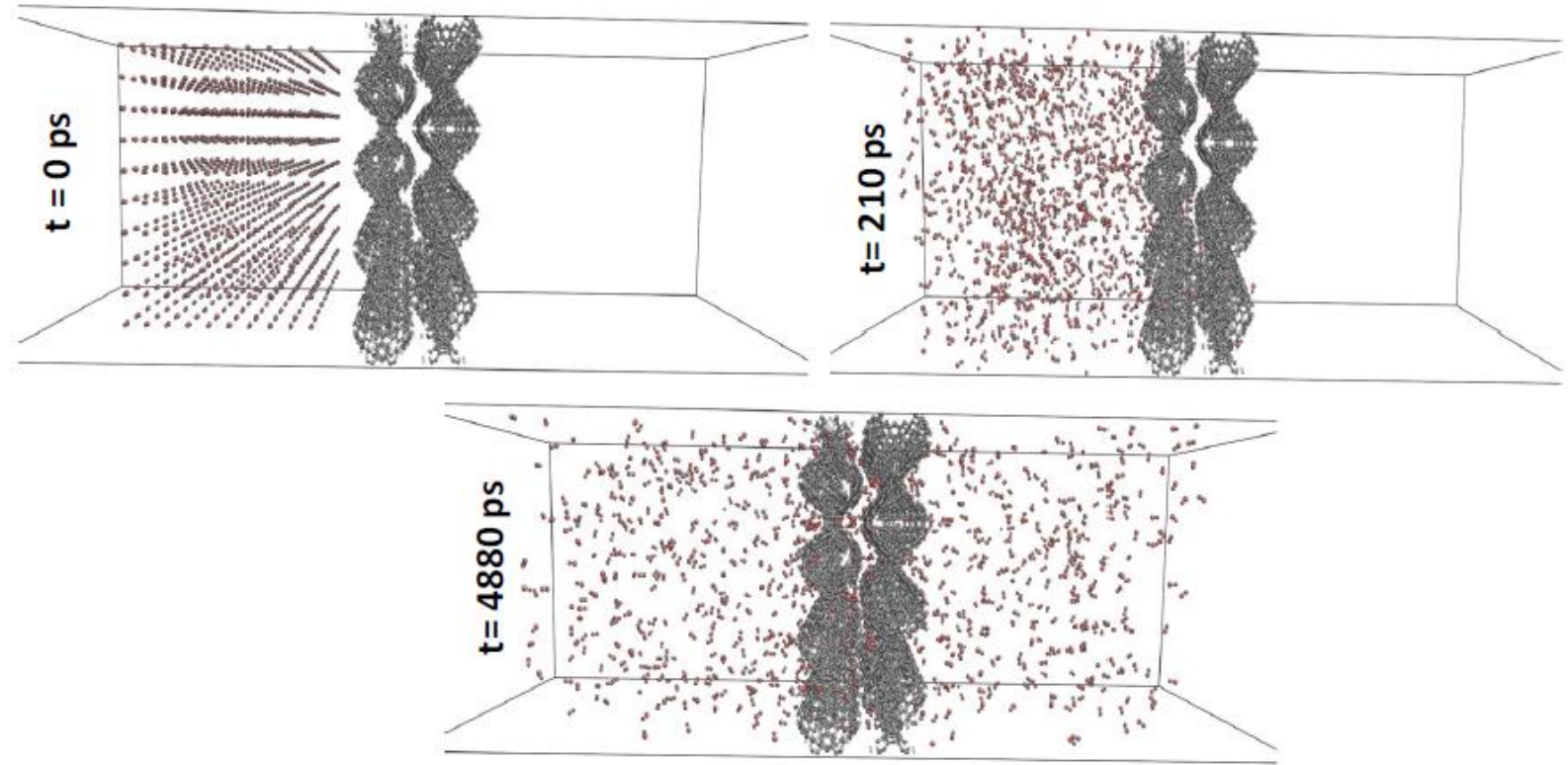

Foram realizados testes usando membranas de duas, três e quatro camadas para cada gás. A Figura 5(a) mostra os resultados obtidos para a taxa de difusão dos gases em função do número de camadas. Pode-se analisar do gráfico que as taxas de difusão são essencialmente determinadas pelo tamanho das moléculas dos gases. Moléculas com diâmetro cinético menor apresentam maiores taxas de transferência, pois tem mais facilidade de atravessar a membrana. Os diâmetros cinéticos para os gases estudados na ordem decrescente são: $\mathrm{CH}_{4}, \mathrm{CO}, \mathrm{N}_{2}, \mathrm{O}_{2}$, $\mathrm{CO}_{2}, \mathrm{H}_{2}$ (Yampolskii et al.,2006). Essa mesma ordem é observada no gráfico das taxas, sendo o $\mathrm{CH}_{4}$ o que apresenta menor taxa de transferência. Isto mostra que o material testado apresenta potencial de uso como peneira molecular, podendo separar correntes de gases pelo seu tamanho. A Figura 5(a) também mostra que a taxa diminui com o aumento do número de camadas, como esperado (a taxa de transferência decai de maneira inversamente proporcional a espessura da membrana (Yampolskii et al.,2006). 
Figura 4 - Exemplo de curvas referentes ao número de moléculas que cruzam a membrana em função do tempo nas simulações. As linhas retas representam as retas obtidas por regressão utilizada para o cálculo das taxas.

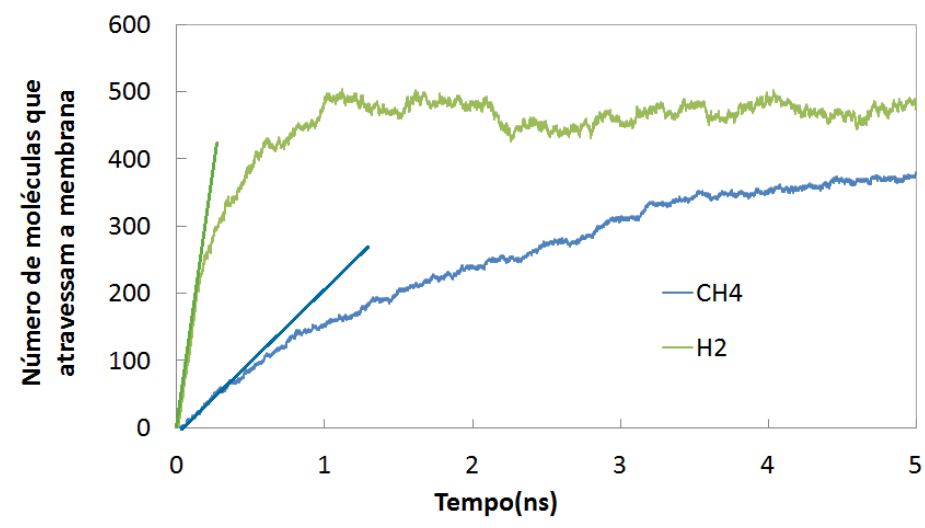

Figura 5 - (a) Taxas de transferência dos gases pela membrana em função do número de camadas de fulerenos porosos empilhadas. (b) Seletividade ideal para diferentes pares de gases em função do número de camadas da membrana.

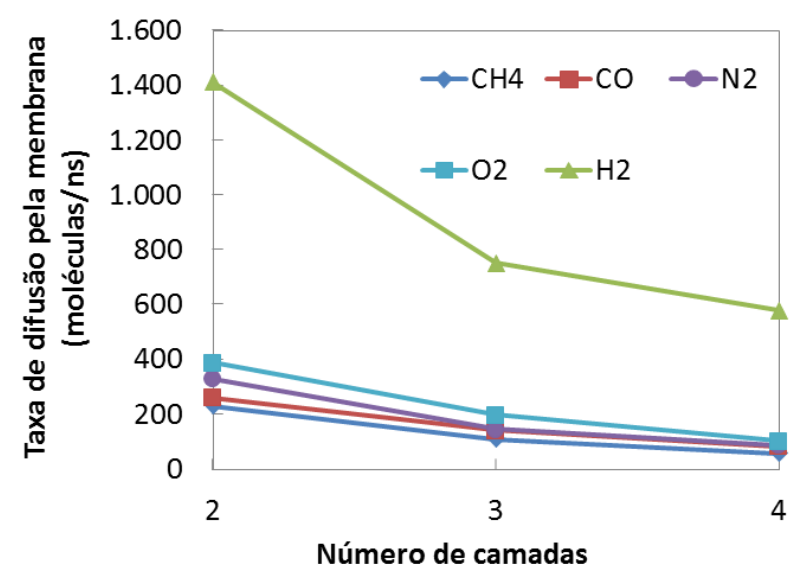

(a)

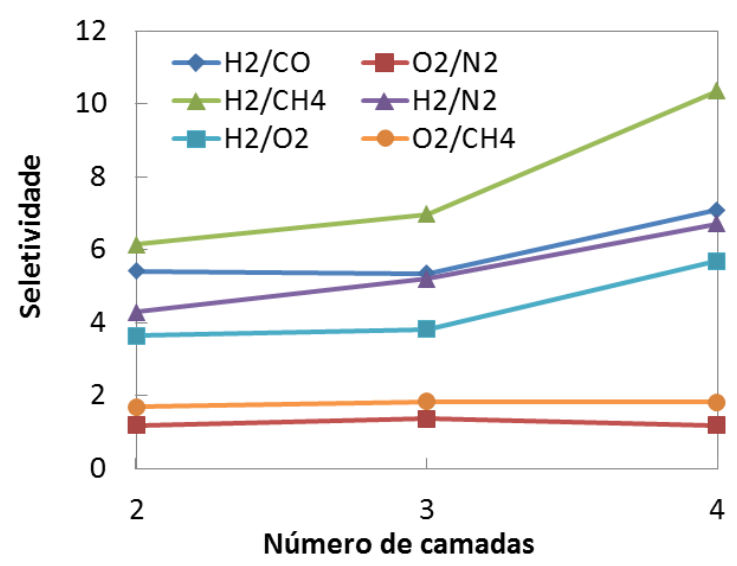

(b)

A partir das taxas de transferência, foram calculadas as seletividades ideais para alguns pares de gases de interesse industrial (por exemplo, $\mathrm{H}_{2} / \mathrm{CO}$ para processos envolvendo gás de síntese, $\mathrm{H}_{2} / \mathrm{CH} 4$ para remoção de hidrogênio do gás natural entre outros). A seletividade ideal é definida como a razão entre as taxas de dois gases:

$$
\text { Seletividade } A / B=\operatorname{Taxa} A / \operatorname{Taxa} B
$$

A figura 5(b) mostra a seletividade ideal de alguns pares de gases em função do número de camadas. Essa figura mostra que essas membranas são seletivas para diversos pares de gases que são importantes em alguns processos industriais, como por exemplo, purificação de correntes $\mathrm{H}_{2} / \mathrm{CO}$ em processos envolvendo gás de síntese e remoção de hidrogênio em gás natural $\left(\mathrm{H}_{2} / \mathrm{CH} 4\right)$, entre outros. Deve-se ressaltar que além desta alta seletividade, o material 
apresenta elevada resistência mecânica e térmica (Kleinpaul et al, 2016), podendo ser aplicado em processos sob altas temperaturas e pressões, o que geralmente não é possível usando as membranas poliméricas tradicionais, por exemplo. Devido a esta maior resistência mecânica, o material poderia ser usado na criação de módulos de membranas mais finos e compactos, potencialmente levando a redução do consumo de energia do processo.

\section{CONCLUSÕES}

Foi analisado o potencial de aplicação de novas estruturas nanoporosas como membranas em processos de separação de gases de grande importância industrial. As simulações mostram que estas membranas apresentam uma seletividade apropriada para aplicação em diversos processos industriais, além de outras propriedades interessantes (resistência mecânica e térmica, distribuição de tamanhos de poro uniformes) demonstradas em um estudo anterior (Kleinpaul et al., 2016). Este trabalho também demonstra a utilidade de simulações de dinâmica molecular para a obtenção de informações essenciais para o desenvolvimento de processos e no estudo de novos materiais de alto desempenho.

\section{REFERÊNCIAS}

CHENG, A.; STEELE, W. A. J. Chem. Phys. 1990, 92, 3858.

JIAO, S.; XU, Z. Selective Gas Diffusion in Graphene Oxides Membranes: A Molecular Dynamics Simulations Study. ACS Appl.Mat.\&Int. 2015, 7, 9052-9059.

JORGENSEN, W. L.; MAXWELL, D. S.; TIRADO-RIVES, Development and Testing of the OPLS All-Atom Force Field on Conformational Energetics and Properties of Organic Liquids. J. Am. Chem. Soc. 1996, 118, 11225-11236.

KLEINPAUl, J.; PAGNUSSATTI, R. A.; ARÊDE, H. R. ; PAUPITZ, R. ; MUNIZ, A. R. Aplicação de Simulação Molecular na Determinação da Estrutura e Propriedades de Nanomateriais Porosos baseados em Carbono. XXI Congresso Brasileiro de Engenharia Química, 2016.

LU, G. Q., ZHAO, X.S. Nanoporous Materials: Science and Engineering. Series on Chemical Engineering, Book 4. World Scientific Publishing Co, 2004.

PAUPITZ, R.; JUNKERMEIER, C. E.; VAN DUIN, A.C.T.; BRANICIO, P.S.; Fullerenes generated from porous structures. Phys. Chem. Chem. Phys. 2014,16, 25515.

PLIMPTON, S. Fast Parallel Algorithms for Short-Range Molecular Dynamics. J. Comp. Phy. 1995.

YAMPOLSKII, Y.; PINNAU, I.;FREEMAN, B. Materials: Science of Membranes for Gas and Vapor Separation. John Wiley \& Sons, Ltd,2006. 